\title{
Investigating the Mechanism of Guizhi Fuling Formula in the Treatment of Primary Dysmenorrhea based on Network Pharmacology and Molecular Docking
}

\section{Lu Sun}

Beijing University of Chinese Medicine Affiliated Dongzhimen Hospital https://orcid.org/0000-00015958-1741

\section{Zining Wang}

Beijing University of Chinese Medicine Affiliated Dongzhimen Hospital

Jian Li

Beijing University of Chinese Medicine

\section{Li Xu}

Beijing University of Chinese Medicine Affiliated Dongzhimen Hospital

Xiaoou Xue ( $\sim$ pro_xue@163.com)

Beijing University of Chinese Medicine Affiliated Dongzhimen Hospital https://orcid.org/0000-0002$1381-0550$

\section{Research}

Keywords: Primary dysmenorrhea, Guizhi Fuling Formula, Network pharmacology, Molecular docking, Target prediction

Posted Date: January 25th, 2021

DOl: https://doi.org/10.21203/rs.3.rs-151904/v1

License: (c) (1) This work is licensed under a Creative Commons Attribution 4.0 International License. Read Full License 


\section{Abstract}

Background: Primary dysmenorrhea(PD)is the most common gynecologic disorder.Despite the prevalence is high, it is often underdiagnosed,undertreated and normalized even by patients themselves. Guizhi Fuling Formula (GFF) is experientially used for the treatment of PD in a long time. Therefore, the efficiency and potential mechanism are waiting to identify.

Methods: We adopted network pharmacology integrated molecular docking strategy

in this study.Based on published literatures, the relative compounds of GFF were selected preliminarily. Secondly, the putative targets of PD were obtained by wide-searching DisGeNET, OMIM, Drugbank and GeneCards databases.With protein-protein interaction(PPI) analysis, GO and KEGG pathway enrichment analysis and molecular docking ,we systematically evaluated the relationship of herb ingredients and disease targets.

Results: The results showed that 30 ingredients of GFF and 43 hub targets made a difference.Under the further analysis,8 targets(EGFR,AKT1,PTGS2,TNF,ESR1,AHR,CTNNB1,CXCL8) were recognized as key therapeutic targets with excellent binding. The enrichment analyses indicated that the GFF had the potential to influence varieties of biological pathways, especially the pathways in cancer and steroid hormone biosynthesis, which play an important part in the pathogenesis of primary dysmenorrhea.

Conclusion: GFF influenced primary dysmenorrhea through the synergistic effect of multiple components, multiple targets, and multiple pathways. This study predicted

the potential mechanism, hope that could made contribution for clinical application and scientific research.

\section{Introduction}

Primary dysmenorrhea (PD) is the most common gynecological disorder, whose definition was epitomized a painful menstruation without the pelvic pathology[1, 2].

There are many studies have demonstrated that PD affect women's academic performance or work management,even the quality of life (QoL), this phenomenon not only bring troubles to people but also have an impact on social and economy[3,4].

The latest PD guideline systematically reviewed the intervention of primary dysmenorrhea.Medical therapy is first-recommended,then the complementary and alternative therapy is also recommended ,surgical management should be considered only the above-mentioned therapies has not been satisfactory[5]. As the first-line drug, NSAIDs were found to be consistently more effective than placebo, although adverse effects were significantly more common.Besides,some RCTs confirmed that cyclic oral contraceptives $(\mathrm{COC})$ was superior to placebo in the treatment of primary dysmenorrhea[6-7]. Recently,Yutaka O.et al.verified the favorable tolerability and efficiency of the long-term use of Dienogest 
for patients with PD, which as a progestin and probably caused irregular uterine bleeding and associated anemia[8].

Compared with those interventions, Traditional Chinese medicine may be able to offer more cost-effective and safe alternative treatments against PD. Guizhi Fuling Formula was initially seen in Synopsis of Golden Chamber(Fig. 1), which is mainly composed of Cinnamomi Ramulus(Guizhi), Poria(Fuling), Moutan Cortex(Danpi), Persicae Semen(Taoren), Peony(Baishao). In the treatment of many gynecological diseases such as hysteromyoma, dysmenorrhea, ovarian cyst etc, it showed ideal curative effect.Evidences on literatures and animal-model trials verified the efficiency of GFF treating PD[9-10].

However,researches about systematic network analysis are deficient comparatively and contribution of the special compatibility of these five herbs on ameliorating PD is not corroborated. Thus, we conducted network pharmacology integrated molecular docking analysis based on the interaction of drugs, diseases, genes, proteins and targets in the biological network to investigate the bioactive constituents and potential mechanism of PD therapy.

\section{Materials And Methods}

\section{Screening of bioactive compounds of GFF}

Traditionally, many previous researches based on Traditional Chinese Medicine System Pharmacology Database [11] (TCMSP, http://lsp.nwu.edu.cn/tcmsp.php), which was not promptly updated.To insure the scientificity of our study, we did not use TCMSP Database but wide-searched related researches on PubMed(https://www.ncbi.nlm.nih.gov/pubmed/)and China National Knowledge Infrastructure Database (CNKI, http://www.cnki.net/) for published chemical constituents of herbs of GFF. Then, downloaded 2D structure from PubChem[12] (https://pubchem.ncbi.nlm.nih.gov/)and used ChemDraw[13] (http://www.chemdraw.com.cn/) for complementary.Finally, based on SwissADME[14] (http://www.swissadme.ch/index.php),we selected bioactive compounds of GFF.

\section{Prediction of putative targets of GFF}

We utilized the SwissTargetPrediction [15] (http://www.swisstargetprediction.ch/) to predict potential targets of GFF compounds. Besides, the UniProt [16] (http://www.uniprot.org/) was applied to convert the protein name of the GFF bioactive compounds to the gene names. We filtered items by "Reviewed"and "Human",then used the result to standardize targets and deleted data duplicates.

\section{Identification of PD related targets}

With a keyword searching of "Primary Dysmenorrhea"among four database: (1) the GeneCards database [17] (https://www.genecards.org/)(2) the Online Mendelian Inheritance in Man database [18] (OMIM, http://omim.org/); (3)the DisGeNET databae[19](http://www.tdisgenet.org/search),(4)the DrugBank 
database [20] (https://www.drugbank.ca/), we gathered related PD genes and made intersection with bioactive compound genes for further analysis.

\section{Protein-protein interaction (PPI) network construction}

Most of the proteins appear to activate their functionalities through their interaction. By evolving core regions with surface residues and hotspot residues at the interface, it could modulate cellular processes such as signal transduction, which provided evidences for drug designing or discovering.[21-22] Thus, we submitted the PD targets and the intersection separately to STRING 11.0 database [23] (https://string$\mathrm{db}$. org/)for PPI analysis, which set filter as"Homo sapiens"organism, to identify important targets in antiPD system.

\section{Network construction}

To characterize the mechanisms of GFF in treating PD from a network target perspective, we employed the Cytoscape 3.8.2[24] (https://cytoscape.org/) to construct visualization networks as follows:(1) Compound-target network; (2) PPI network of PD targets; (3) PPI network of compound PD targets; (4) Compound-target-pathway network. When analyzed the topological properties of the PPI network by the Cytoscape,there were three indices recommended to assess nodes and edges. Firstly and commonly, we used "Degree” to evaluated the interaction between nodes [25]. Secondly,"Betweenness Centrality”was applied to determined the amount of information passing between nodes[26].Thirdly, "Closeness Centrality" reflects the closeness of a node to other nodes[27]. The higher values of this three parameters are, the more important the nodes are. Thus, the hub genes for molecular docking were selected systematically with the help of above parameters.

\section{Functional enrichment analysis}

As same as the choosing principle of bioactive compounds, Functional Annotation tool of Database for Annotation,Visualization and Integrated Discovery (DAVID) 6.8 [28] (https://david.ncifcrf.gov/) was not the best candidate, whose speed of updating is too sluggish. What's more,Metascape[29] (https://metascape.org/)(https://david.ncifcrf.gov/) are recommended to conducted GO functional analysis and KEGG pathways analysis recently. We set parameters as follow: Overlap $>2, P$ value $>0.01$ and Enrichment>1.5.

\section{Molecular docking simulation}

This study selected the" component target-pathway "network moderate value (degree) top 8 target protein,and these eight potential targets with nine corresponding compounds were simulated by molecular docking.Initially, downloading the PDB format of the 3D structure of the target protein from the Protein Data Bank database[30] (https://www.rcsb.org/). The screening conditions were set as follows: (1) the organism comes from Homo sapiens.(2) the protein structure is obtained by X-crystal diffraction;

(3) the crystal resolution of the protein is less than $2 \AA$; (4) preferential selection of protein structures reported in the literature of molecular docking. And the mol2 format of the 3D structure of the compounds 
were downloaded in the ZINC database[31] (https://zinc.docking.org/).Secondly,the Pymol 2.4[32] was used to remove water molecules and raw ligands.After, utilizing AutoDockTools1.5.6[33] (http://mgltools.scripps.edu/documentation/links/autodock) to hydrogenate and charge,and then the $\mathrm{pdb}$ format of compounds and core protein were converted into pdbqt format and active pocket was found. Eventually,Running AutoDock Vina1.1.2[34] with CMD command characters for molecular docking and calculating binding energy.Visualization of results using PyMOL 2.4 software.

\section{Results}

\section{Compound-target network}

Based on the Pharmacokinetics and Druglikeness parameters in SwissADME[14], we selected a total of 30 candidate bioactive compounds.(Complementary Table 1). Then, every compound was submitted on Swiss Prediction for identifying related targets. There were 374 compound- predicted targets of GFF made up the network, which consist of 409 nodes and 907 edges(Fig. 3). We found that a single target can be co-regulated by a variety of compounds to trigger the biological effects, which may play a vital role in treating PD.

\section{PPI network of PD targets}

Integrated targets which retrieved from GeneCards, DisGeNet, OMIM and DrugBank database, then we uploaded them to the STRING database and constructed a PPI network with 319 nodes and 4270 edges. For better visualization,the Cytoscape was applied for network mapping. Based on analyzing network, we used degree(value>2) cutting low-related targets.From yellow to blue, the degree was increasing. (Fig.4)The bigger the node was, the higher the degree was.

\section{PPI network of compound PD targets}

We made a intersection between GFF bioactive targets and PD related targets,

43 targets were identified as the potential targets for treating PD(Fig.5a). Similarly, we conducted a PPI network to explore the mechanism of the drugs in disease therapy and discover the interactive effects. Then employed Cytoscape to analyze the topological feature of the PPI network and cut low-related targets, 39 genes arranged in a concentric circle according to the degree.(Fig.5b) According to average degree(value $>17$ ), Betweenness Centrality $(>0.03)$ and Closeness Centrality $(>0.06)$, we selected a total of 8 hub genes(Table 1),including EGFR,AKT1,PTGS2,TNF,ESR1,AHR,CTNNB1,CXCL8. These hub genes are of significance in the network, which would be more suitable for further molecular docking analysis.

\section{Table1.Topological information of 8 hub genes}




\begin{tabular}{ccccc}
\hline Gene name & Protien name & BetweennessCentrality & ClosenessCentrality & Degree \\
\hline EGFR & Epidermal growth factor receptor & 0.079120463 & 0.701754386 & 23 \\
AKT1 & RAC-alpha serine/threonine-protein kinase & 0.077033148 & 0.701754386 & 23 \\
PTGS2 & Prostaglandin G/H synthase 2 & 0.074264862 & 0.689655172 & 23 \\
TNF & Tumor necrosis factor & 0.10058786 & 0.689655172 & 23 \\
ESR1 & Estrogen receptor & 0.062011048 & 0.677966102 & 23 \\
AHR & Aryl hydrocarbon receptor & 0.042161256 & 0.64516129 & 19 \\
CTNNB1 & Catenin beta-1 & 0.035301494 & 0.625 & 18 \\
CXCL8 & Interleukin-8 & 0.032675776 & 0.634920635 & 18 \\
\hline
\end{tabular}

\section{GO and KEGG enrichment analysis}

The GO items and KEGG pathways are selected based on counts of hit

genes and $\mathrm{P}$ values (Fig. 6). For biological processes, it can be found that the targets were mainly enriched in cellular response to organic cyclic compound(G0:0071407)or nitrogen compound(GO:1901699),steroid metabolic process(G0:0008202),membrane raft(GO:0045121)and oxidoreductase activity(G0:0016705)

For pathway analysis, most involved in pathways in cancer(hsa05200),proteoglycans in cancer(ko05205), human cytomegalovirus infection(hsa05163) and steroid hormone biosynthesis(ko00140).

\section{Molecular docking analysis}

As far as current research is concerned, we choose 8 hub genes and 9 core bioactive compounds(Table 2)to test the potential interaction activity by molecular docking verification. A total of 72 pairs were used for molecular docking simulation.The smaller the value of docking affinity,the more easily the active ingredient binds to the receptor. According to the results of docking, the great majority of binding complexes possessed high binding affinity with an average of $-7.5 \mathrm{kcal} / \mathrm{mol}$. Molecular docking affinity values $<-7$ accounted for $69.44 \%$, indicating better binding activity. The first eight binding complexes with affinity less than -9 were selected and displayed in Table 3,including EGFR-ellagic acid docking (-9.4 $\mathrm{kcal} / \mathrm{mol})$, AHR-taxifolin docking $(-10.3 \mathrm{kcal} / \mathrm{mol})$, AHR-(+)-catechin docking $(-10.2 \mathrm{kcal} / \mathrm{mol})$, AHRbaicalein $(-10.2 \mathrm{kcal} / \mathrm{mol})$, CTNNB1-Stigmasterol docking $(-10.3 \mathrm{kcal} / \mathrm{mol})$, CTNNB1- ellagic acid docking $(-9.9 \mathrm{kcal} / \mathrm{mol})$, CTNNB1-baicalein docking(-9.4 kcal/mol), CTNNB1-(+)-catechin docking $(-9.3 \mathrm{kcal} / \mathrm{mol})$.

\section{Table 2. Topological information of 9 core bioactive compounds}




\begin{tabular}{lcccr}
\hline Molecule name & MOLID & BetweennessCentrality & ClosenessCentrality & Degree \\
\hline quercetin & MOL000098 & 0.660565978 & 0.57208238 & 145 \\
kaempferol & MOL000422 & 0.141343038 & 0.411861614 & 56 \\
beta-sitosterol & MOL000358 & 0.111475745 & 0.390015601 & 38 \\
baicalein & MOL002714 & 0.096768005 & 0.374812594 & 34 \\
Stigmasterol & MOL000449 & 0.090225666 & 0.382848392 & 30 \\
hederagenin & MOL000296 & 0.055000783 & 0.372578241 & 24 \\
ellagic acid & MOL001002 & 0.031733698 & 0.354609929 & 18 \\
taxifolin & MOL004576 & 0.026248081 & 0.341997264 & 11 \\
$(+)$-catechin & MOL000492 & 0.016160162 & 0.353606789 & 11 \\
\hline
\end{tabular}

\section{Table 3.Analysis of molecular docking results}

\begin{tabular}{lccccc}
\hline N0 & Proteins & PDB ID & Protein structure & Test compounds & Affinity $(\mathrm{kcal} / \mathrm{mol})$ \\
\hline 1 & EGFR & 6Q7D & & ellagic acid & -9.4 \\
& & & &
\end{tabular}

2 AHR 4LBS $\begin{array}{ll}\text { taxifolin } & -10.3 \\ & \end{array}$

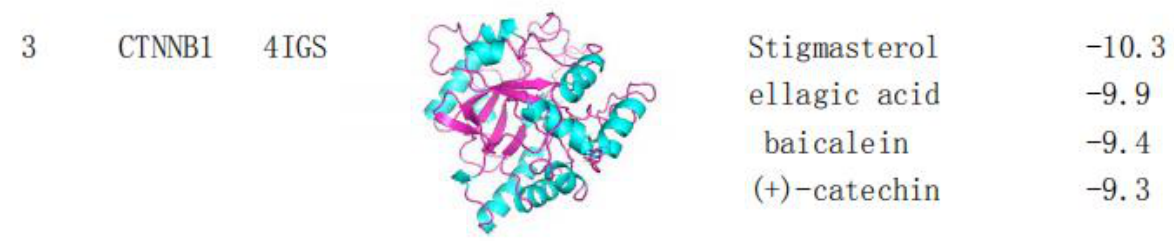

For further explanation, Give an example of AHR-taxifolin docking ,small molecule ligand taxifolin could possibly fit into the active interface pocket formed by interaction amino acid residues in protein (Fig.7). It displayed that a hydrogen bond formation between ligand and residues invloved in TRP- 
20.Consequently,taxifolin stably binded to AHR protein bonds through interaction forces.on account of this experiment,the results of molecular docking showed that the core compounds and key targets screened by network pharmacology had fairly binding activity.

\section{Compound-target -pathway network}

For further exploring the interaction among pathway, gene and compound, Cytoscape was applied again to construct a compound-target-pathway network(Fig.8a).

Based on KEGG enrichment analysis, we obtained 14 pathways which related different targets. Thanks to compound-target network above, the new network was responsibly easy to conducted. As shown in Fig.8a, there were a total of 72 nodes and 187 edges. 14 pathways were interacted with 31 targets and 27 compounds. Subsequently, degree was adopted as the threshold values to identify the significant compounds, targets and pathways. Six compounds, 20 targets and 8 pathways were selected for a more detailed explanation of the associations described above, which displayed in the sub-network(Fig.8b) .

\section{Discussion}

From past to now, the difference between Traditional Chinese Medicine(TCM) and Modern Medicine is the concept of whole and part. The principle of choosing Chinese herbs was based on four properties, five flavors and channel tropism[35].Appropriate compatibility made prognosis of disease better, it was hard to explain concretely the interplay among those herbs for people who never learned about TCM. Thus, we need a evidence-based and illustrative methodology to explore the mechanism.

According to a series of analysis above, we gained six significant compounds, keampferol concentrated in Baishao(Peony), quercetin and isorhamnetin were enriched in Danpi(Moutan Cortex),cerevisterol and 3,5-Dihydroxyergosta-7,22-dien-80-one were found in Fuling(Poria), glycosmisic acid existed in Guizhi(Cinnamomi Ramulus).

Keampferol and quercetin always appeared at the same time due to the familiar effectiveness in antiinflammatory, antioxidation and proapoptosis[36-39].In a network study of TCM treating cancer-related pain[40], quercetin and kaempferol also worked as very important compounds, which could regulate the phosphoinositide 3-kinase (PI3K)/protein kinase B (AKT)pathways to achieve the purpose of antitumor and alleviating pain.It might provide evidences for the effect of quercetin and kaempferol ameliorating the pain of menstruation. Cerevisterol and 3,5-Dihydroxyergosta-7,22-dien-80-one were usually isolated and identified from the mycelia, which could treat inflammatory diseases by targeting an MAPK, NF-KB, AP-1, and Nrf2-mediated HO-1 signaling cascade[41].

Epidermalgrowth factor receptor(EGFR) belongs to a family of four homologous tyrosine kinase receptors (TKRs). This family are one of the major drivers of cancer and is involved in several of the most frequent malignancies such as non-small cell lung cancer, breast cancer, colorectal cancer and ovarian cancer[42]. Although EGFR related to many diseases, there were not published trials confirmed the association 
between EGFR and PD. RAC-alpha serine/threonine-protein kinase \AKT1囚 had a close relationship with gynecologic disease, such as endometriosis[43] and endometrial carcinoma[44]. In our study, it presented a significant relevance to PD, which could provide evidences for further studies.Prostaglandin $\mathrm{G} / \mathrm{H}$ synthase 2ХPTGS2囚is an inducible prostaglandin enzyme whose derivative(PGE2 and PGI2) are the major mediators in decidualization[45].Immunohistochemical tests, western blotting (WB) assays and polymerase chain reaction (PCR)show that PTGS2 expression in the uterine myometrium and glands is directly related to organ inflammation intensity[46]. One pathological mechanism of PD is the increase of PGE2 and PGF2a[47], verifying the great effect of PTGS2 on PD.

The results of pathway analysis suggested that Pathways in cancer, Proteoglycans in cancer, Human cytomegalovirus infection and Steroid hormone biosynthesis showed significant association with PD.It is worth thinking about its close relationship with cancer-related pathways.

Steroid hormone biosynthesis(Complementary Fig.1) contributed to regulate metabolism and stress response, initiate and maintain sexual differentiation and reproduction[48].Follicle-stimulating hormone (FSH) controls the progesterone synthesis from granulosa cells via G protein/adenylate cyclase (CAMP)/protein kinase A (PKA)/MAPK pathway, then progesterone transforms to estrogen under the regulation of enzymes in the steroid hormone metabolism pathway[49]. Thus, it indirectly confirmed the effectiveness of drugs or herbs therapy via hormone pathway. Ji et al. revealed the enhanced steroid hormone biosynthesis during decidualization, which was influenced by metabolic alteration[50].Serotonergic synapse is widely associated with mood disorders, especially in women[51].Thus, PD caused by stress, depression or other mood disorders could treat in this train of thoughts.

Studies about the Adipocytokine signaling pathway and PPAR signaling pathway are limited. Hu et al.corroborated that PPAR signaling pathway participated in the regulation of inflammatory response[52].Dotun et al.indicated that the downregulation of several key genes in the KEGG Adipocytokine Pathway was linked to insulin resistance or inflammation[53].

Overall, it was speculated that the main compositions of GFF may play a significant role in the treatment of PD through hub targets in these top-ranking signaling

pathways. However, some limitations of our study should be considered. For instance, the results are only based on screening already known chemical constituents of GFF,

related targets, and signaling pathways from literatures and existing databases. Consequently, more indepth researches are required for characterization of the underlying mechanisms.

\section{Conclusion}

In the present study, there were four networks that we derived to reveal the significant compounds,targets and pathways of GFF in PD treatment, including compound-target network, PPI network of PD targets, PPI 
network of compound-PD targets and compound-target -pathway network.After sieving and consolidating data from available databases, we got 30 bioactive compounds of GFF and 43 potential targets for treating PD. According to the PPI network, 8 hub genes were highly connected and could be defined as vital targets in the process of treating PD with GFF. In GO and KEGG pathway enrichment analysis, we found that these hub targets ameliorated PD by participating in signal transduction, hormone-metabolism system and inflammatory response. In short, the above results preliminarily predicted the potential mechanism of GFF against PD, visualizing the characteristics of Chinese herbs interaction, which providing more possibility of PD therapy.Actually,further extensive researches such as animal experiments and clinical investigations will be needed, which is necessary to provide more valuable evidences for the application of GFF in treating PD.

\section{Declarations}

\section{Availability of data and materials}

All data in the manuscript are available and they are exhibited in figures and tables.

\section{Acknowledgement}

The authors wish to thank the editor and the anonymous reviewers whose constructive comments are very helpful in strengthening the presentation of this paper.

\section{Author information}

\section{Affiliations}

Department of Gynecology, Beijing University of Chinese Medicine Affiliated Dongzhimen Hospital, No.5 Haiyuncang, Dongcheng District, Beijing, 100700, China.

Lu Sun, Zi-ning Wang, Li Xu, Xiao-ou Xue

School of Chinese Medicine, Beijing University of Chinese Medicine, No.11 Beisanhuandong Road, Chaoyang District Beijing, 100029, China.

Jian Li

\section{Contributions}

SL,WZN and XL: study design; acquisition of data; analysis of data; drafting of the manuscript. LJ and XXO: revision of the manuscript and study supervision. All the author(s) read and approved the final manuscript.

\section{Corresponding authors}

Correspondence to Xiao-ou Xue and Lu Sun. 


\section{Ethics declarations}

\section{Ethics approval and consent to participate}

No applicable.

\section{Competing interests}

Authors declare no conflict of interest.

\section{References}

[1] ACOG Committee opinion No. 760:dysmenorrhea and endometriosis in the adolescent. Obstet Gynecol. 2018;132(6):e249-e258.

[2] Matthewman, G.; Lee, A.; Kaur, J.G.; Daley, A.J. Physical activity for primary dysmenorrhea: A systematic review and meta-analysis of randomized controlled trials. Am. J. Obstet. Gynecol. 2018, 219, 255, doi:10.1016/j.ajog.2018.04.001.

[3] Hailemeskel, S.; Demissie, A.; Assefa, N. Primary dysmenorrhea magnitude, associated risk factors, and its effect on academic performance: Evidence from female university students in Ethiopia. Int. J. Womens Health 2016, 8, 489-496, doi:10.2147/IJWH.S112768.

[4] Elia F.M.,María D.O.Z.,María L. P.F.The impact of dysmenorrhea on quality of life among Spanish female university students.Int. J. Environ. Res. Public Health 2019, 16, 713; doi:10.3390/ijerph16050713 [5] Burnett M, Lemyre M.No. 345-Primary Dysmenorrhea Consensus Guideline.J Obstet Gynaecol Can 2017;39(7):585-595

[6] Harada T, Momoeda M, Terakawa N, et al. Evaluation of a low-dose oral contraceptive pill for primary dysmenorrhea: a placebo-controlled, double-blind, randomized trial. Fertil Steril 2011;95:1928-31.

[7] Dmitrovic R, Kunselman AR, Legro RS. Continuous compared with cyclic oral contraceptives for the treatment of primary dysmenorrhea: a randomized controlled trial. Obstet Gynecol 2012;119:1143-50.

[8] Osuga Y, Hayashi K, Kanda S. Long-term use of dienogest for the treatment of primary and secondary dysmenorrhea. Journal of Obstetrics and Gynaecology Research.2020; 46,4.doi:10.1111/jog.14209.

[9] Wei Zhang, Dianrong Song et al.Literature research and status analysis on clinical application of Guizhi Fuling Formula .China Journal of Chinese Materia Medica.https://doi.org/10.19540/j.cnki.cjcmm.20200615.501.

[10] Yezhe Cheng , Yanjie Chu , Xitong Su et al. Pharmacokinetic Pharmacodynamic modeling to study the anti-dysmenorrhea effect of Guizhi Fuling capsule on primary dysmenorrhea rats, Phytomedicine (2018), doi: 10.1016/j.phymed.2018.04.041 
[11] Ru J, Li P, Wang J, Zhou W, Li B, Huang C, et al. TCMSP: a database of systems pharmacology for drug discovery from herbal medicines. J Chem. 2014;6:13.

[12] Kim S, Thiessen PA, Bolton EE, Chen J, Fu G, Gindulyte A, Han L, He J, He S, Shoemaker BA, Wang J, Yu B, Zhang J, Bryant SH. PubChem substance and compound databases. Nucleic Acids Res. 2016;44(D1):D1202-13.

[13] Evans DA. History of the Harvard ChemDraw project. Angew Chem Int Ed Engl. 2014;53(42):11140-5.

[14] SwissADME: a free web tool to evaluate pharmacokinetics, drug-likeness and medicinal chemistry friendliness of small molecules. Sci. Rep. (2017) 7:42717.

[15] Gfeller D, Grosdidier A, Wirth M, Daina A, Michielin O, Zoete V. SwissTargetPrediction: a web server for target prediction of bioactive small molecules. Nucleic Acids Res. 2014;42:W32-8.

[16] The UniProt Consortium.UniProt: a worldwide hub of protein knowledge.Nucleic Acids Res. 47: D506515 (2019)

[17] Stelzer G, Rosen N et al. The GeneCards Suite: From Gene Data Mining to Disease Genome Sequence Analyses.Curr Protoc Bioinformatics. 2016 Jun 20;54:1.30.1-1.30.33. doi: 10.1002/cpbi.5.

[18] Amberger JS, Hamosh A et al. Searching Online Mendelian Inheritance in Man (OMIM): A Knowledgebase of Human Genes and Genetic Phenotypes.Curr Protoc Bioinformatics. 2017 Jun 27;58:1.2.1-1.2.12. doi: 10.1002/cpbi.27.

[19] Piñero J, Bravo À, Queralt-Rosinach N et al. DisGeNET: a comprehensive platform integrating information on human disease-associated genes and variants. Nucleic Acids Res. 2017;45(D1):D833-9

[20] Wishart DS, Feunang YD, Guo AC et al. DrugBank 5.0: a major update to the DrugBank database for 2018. Nucleic Acids Res. 2017 Nov 8. doi: 10.1093/nar/gkx1037.

[21] Athanasios A, Charalampos V, Vasileios T, Ashraf GM.Protein-Protein Interaction (PPI) Network: Recent Advances in Drug Discovery.Curr Drug Metab. 2017;18(1):5-10. doi: 10.2174/138920021801170119204832.

[22] Murakami Y, Tripathi LP, Prathipati P, Mizuguchi K. Network analysis and in silico prediction of protein-protein interactions with applications in drug discovery.Curr Opin Struct Biol. 2017 Jun;44:134142. doi: 10.1016/j.sbi.2017.02.005.

[23] Szklarczyk D, Morris JH, Cook H, Kuhn M, Wyder S, Simonovic M, et al. The STRING database in 2017: quality-controlled protein-protein association networks, made broadly accessible. Nucleic Acids Res. 2017;45(D1):D362-8. 
[24] Doncheva NT, Morris JH, Gorodkin J, Jensen LJ. Cytoscape StringApp: Network Analysis and Visualization of Proteomics Data.J Proteome Res. 2019 Feb 1;18(2):623-632. doi:

10.1021/acs.jproteome.8b00702.

[25] Lawyer G.Understanding the influence of all nodes in a network.Sci Rep. 2015 Mar 2;5:8665. doi: $10.1038 /$ srep08665.

[26] Durón C, Pan Y, Gutmann DH, Hardin J, Radunskaya A.Variability of Betweenness Centrality and Its Effect on Identifying Essential Genes.Bull Math Biol. 2019 Sep;81(9):3655-3673. doi: 10.1007/s11538018-0526-z.

[27] Beseler CL, Keeney AJ, Garratt R.Assessing Connections in an Agricultural Community Using Social Network Analysis.J Agromedicine. 2020 Nov 14:1-11. doi: 10.1080/1059924X.2020.1837317.

[28] Zhou et al., Nature Communication (2019), 10(1):1523.

[29] Zhou Y, Zhou B, Pache $L$ et al. Metascape provides a biologist-oriented resource for the analysis of systems-level datasets. Nat Commun. 2019 Apr 3;10(1):1523. doi:10.1038/s41467-019-09234-6.

[30] Chakraborty S, Phu M, de Morais TP et al.The PDB database is a rich source of alpha-helical antimicrobial peptides to combat disease causing pathogens.F1000Res. 2014 Dec 5;3:295. doi: 10.12688/f1000research.5802.2.

[31] Koes DR, Camacho CJ.ZINCPharmer: pharmacophore search of the ZINC database.Nucleic Acids Res. 2012 Jul;40(Web Server issue):W409-14. doi: 10.1093/nar/gks378.

[32] Rigsby RE, Parker $A B$. Using the PyMOL application to reinforce visual understanding of protein structure.Biochem Mol Biol Educ. 2016 Sep 10;44(5):433-7. doi: 10.1002/bmb.20966.

[33] Nehme El-Hachem, Benjamin Haibe-Kains et al.AutoDock and AutoDockTools for Protein-Ligand Docking: Beta-Site Amyloid Precursor Protein Cleaving Enzyme 1(BACE1) as a Case Study.Methods Mol Biol. 2017;1598:391-403.doi:10.1007/978-1-4939-6952-4_20.

[34] Valdés-Tresanco MS, Valdés-Tresanco ME, Valiente PA.AMDock: a versatile graphical tool for assisting molecular docking with Autodock Vina and Autodock4.Biol Direct. 2020 Sep 16;15(1):12. doi: 10.1186/s13062-020-00267-2.

[35] Zhong LY, Cui MN, Yang M et al. Modern researches on effect of processing of Chinese herb medicine on Chinese medical properties. Zhongguo Zhong Yao Za Zhi. 2019 Dec;44(23):5109-5113. Chinese. doi: 10.19540/j.cnki.cjcmm.20190916.301.

[36] Tian Chunlian, Liu Xin, Chang Yu, et al. Investigation of the anti-inflammatory and antioxidant activities of luteolin, kaempferol, apigenin and quercetin. 2021, 137:257-264. 
[37] Zhu S, Wang H, Zhang J, Yu C, Liu C, Sun H, et al. Antiasthmatic activity of quercetin glycosides in neonatal asthmatic rats. 3 Biotech. 2019;9(5):189.

[38] Yu KH, Lee Cl. Quercetin Disaggregates Prion Fibrils and Decreases Fibril-Induced Cytotoxicity and Oxidative Stress.Pharmaceutics. 2020 Nov 11;12(11):E1081. doi: 10.3390/pharmaceutics12111081.

[39] Jia Z, Chen A, Wang C, He M, Xu J, Fu H, et al. Amelioration effects of Kaempferol on immune response following chronic intermittent cold-stress. Res Vet Sci. 2019;125:390-6.

[40] Chang J, Liu L, Wang Y.Investigating the Multitarget Mechanism of Traditional Chinese Medicine Prescription for Cancer-Related Pain by Using Network Pharmacology and Molecular Docking Approach.Evid Based Complement Alternat Med. 2020 Nov 10;2020:7617261. doi:

10.1155/2020/7617261. eCollection 2020.

[41] Alam MB, Chowdhury NS, Sohrab MH.Cerevisterol Alleviates Inflammation via Suppression of MAPK/NF-kappaB/AP-1 and Activation of the Nrf2/HO-1 Signaling Cascade.Biomolecules. 2020 Jan 29;10(2):199. doi: 10.3390/biom10020199.

[42] Zanetti-Domingues LC, Bonner SE. Mechanisms of Action of EGFR Tyrosine Kinase Receptor Incorporated in Extracellular Vesicles.Cells. 2020 Nov 19;9(11):2505. doi: 10.3390/cells9112505.

[43] Feng W-Q, Deng Y-X, Ma Y.Expression of miR-34a-5p and AKT1 gene in endometrial tissue of endometriosis and its effect on migration and invasion of endometrial stromal cells.Med $\mathrm{J}$ Chin PLA. 2020,45(5):503-508.

[44] Shi QQ, Li JX, Liu Y. To investigate the expression and clinical significance of miR-183 and Akt1 protein in endometrial carcinoma[ J] . Chin J Front Med(Electron Ed), 2018, 10(2): 37-40.

[45] Isayama Keishiro, Zhao Lijia, Chen Huatao, et al. Removal of Rev-erba inhibition contributes to the prostaglandin G/H synthase 2 expression in rat endometrial stromal cells.2015, 308(8):E650-61.

[46] Xiao-Hong Li, Yan-Ru Liu, Da-Hai Jiang, et al. Research on the mechanism of Chinese herbal medicine Radix Paeoniae Rubra in improving chronic pelvic inflammation disease by regulating PTGS2 in the arachidonic acid pathway. 2020, 129

[47] Liedman, R.; Hansson, S.R.; Howe, D.; Igidbashian, S.; Mcleod, A.; Russell, R.J.; Åkerlund, M. Reproductive hormones in plasma over the menstrual cycle in primary dysmenorrhea compared with healthy subjects. Gynecol. Endocrinol. 2008, 24, 508-513.

[48] Lina Schiffer, Lise Barnard, Elizabeth S. Baranowski, et al. Human steroid biosynthesis, metabolism and excretion are differentially reflected by serum and urine steroid metabolomes: A comprehensive review. 2019, 194. 
[49] Huanxian Cui, Guiping Zhao, Jie Wen, et al. Follicle-stimulating hormone promotes the transformation of cholesterol to estrogen in mouse adipose tissue.. 2018, 495(3):2331-2337.

[50] Ji-Long Liu, Wen-Qian Zhang, Miao Zhao, et al. Integration of Transcriptomic and Metabolomic Data Reveals Enhanced Steroid Hormone Biosynthesis in Mouse Uterus During Decidualization. 2017, 17(19).

[51] Alana Castro Panzenhagen, Renata Basso Cupertino, Bruna Santos, et al. GENE-SET ANALYSIS OF SEROTONERGIC SYNAPSE GENES IN ADULTHOOD ADHD. 2019, 29:S823-S823.

[52] Hu T, Lin Q, Guo T et al. Polysaccharide isolated from Phellinus linteus mycelia exerts antiinflammatory effects via MAPK and PPAR signaling pathways,Carbohydrate Polymers(2018), https://doi.org/10.1016/j.carbpol.2018.08.021.

[53] Dotun Ogunyemi, Jun Xu, Arnold M. Mahesan, et al. Differentially expressed genes in adipocytokine signaling pathway of adipose tissue in pregnancy. 2013, 3(2):86-95.

\section{Figures}

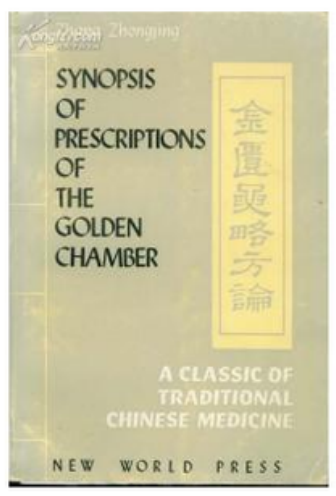

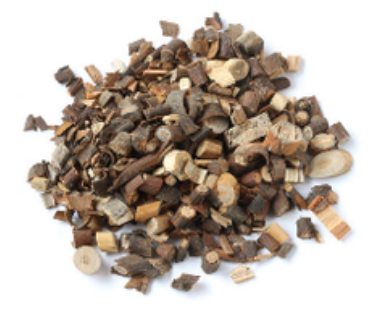

Guizhi

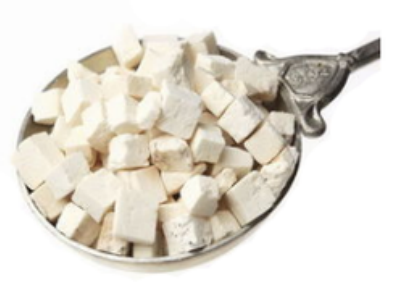

Fuling

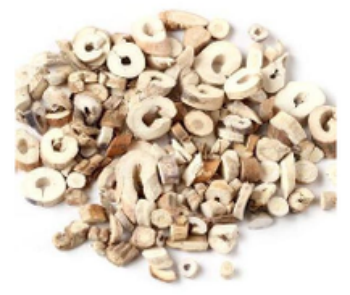

Danpi

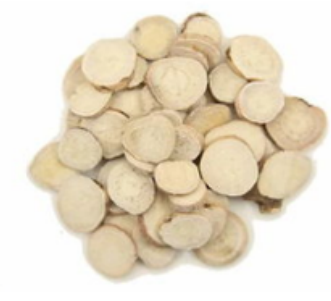

Baishao

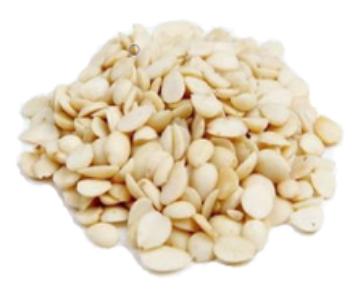

Taoren

\section{Figure 1}

Synopsis of Golden Chamber and Guizhi Fuling Formula 


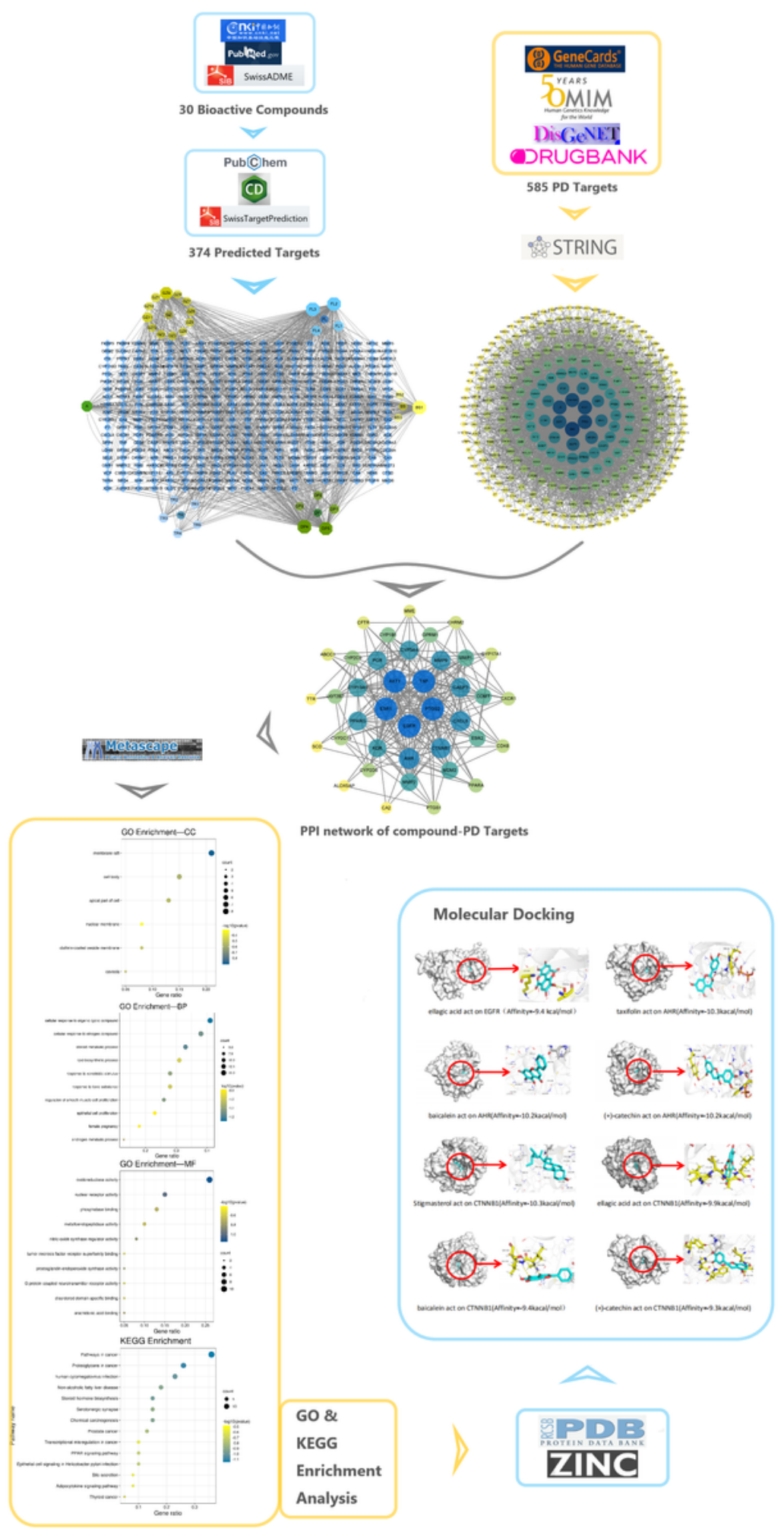

\section{Figure 2}

Flowchart of investigating the mechanism of GFF in PD treatment 


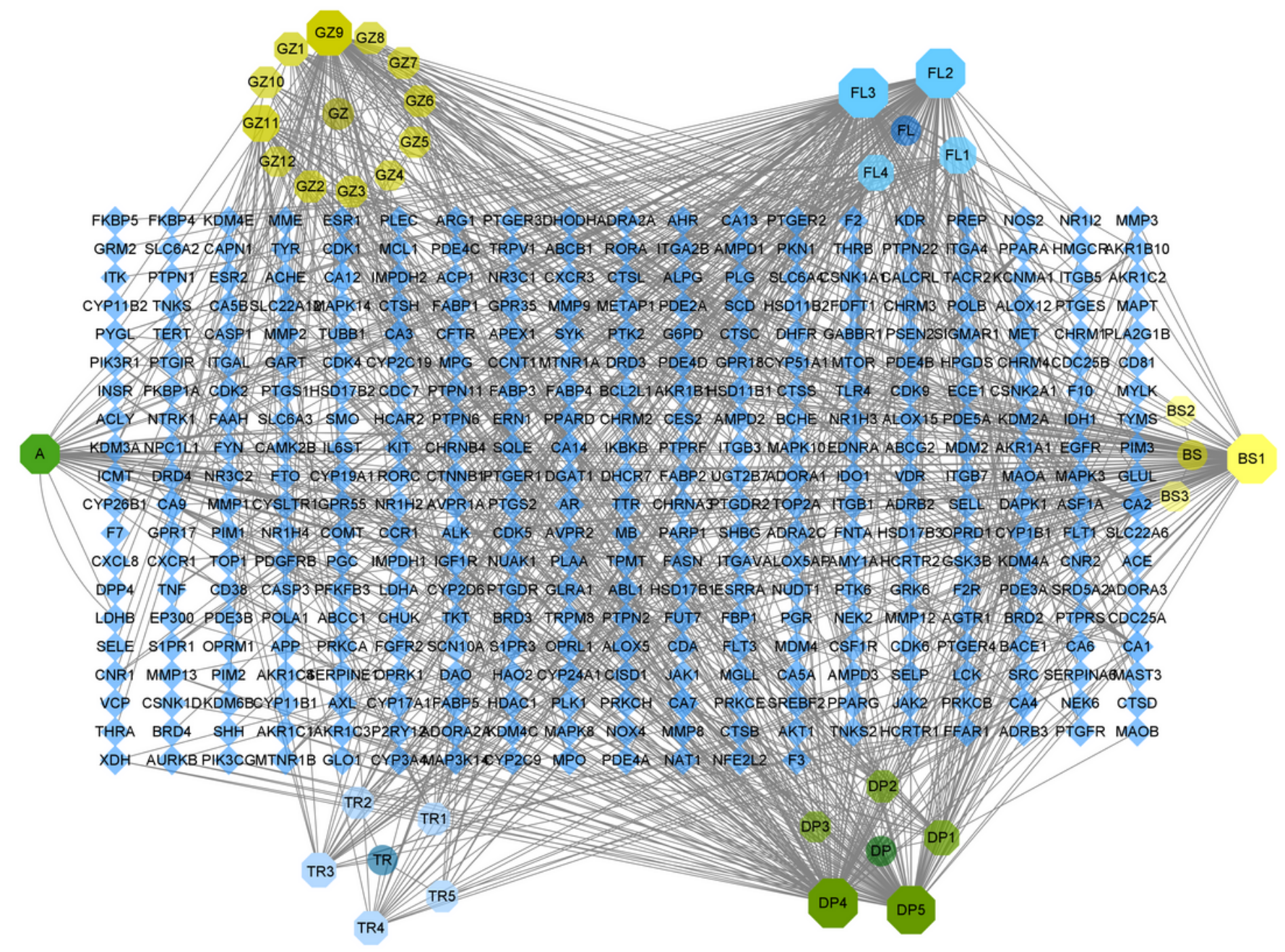

\section{Figure 3}

Compound-target network. Colourful octagen nodes represent bioactive compounds of GFF and blue diamond nodes represent corresponding targets 


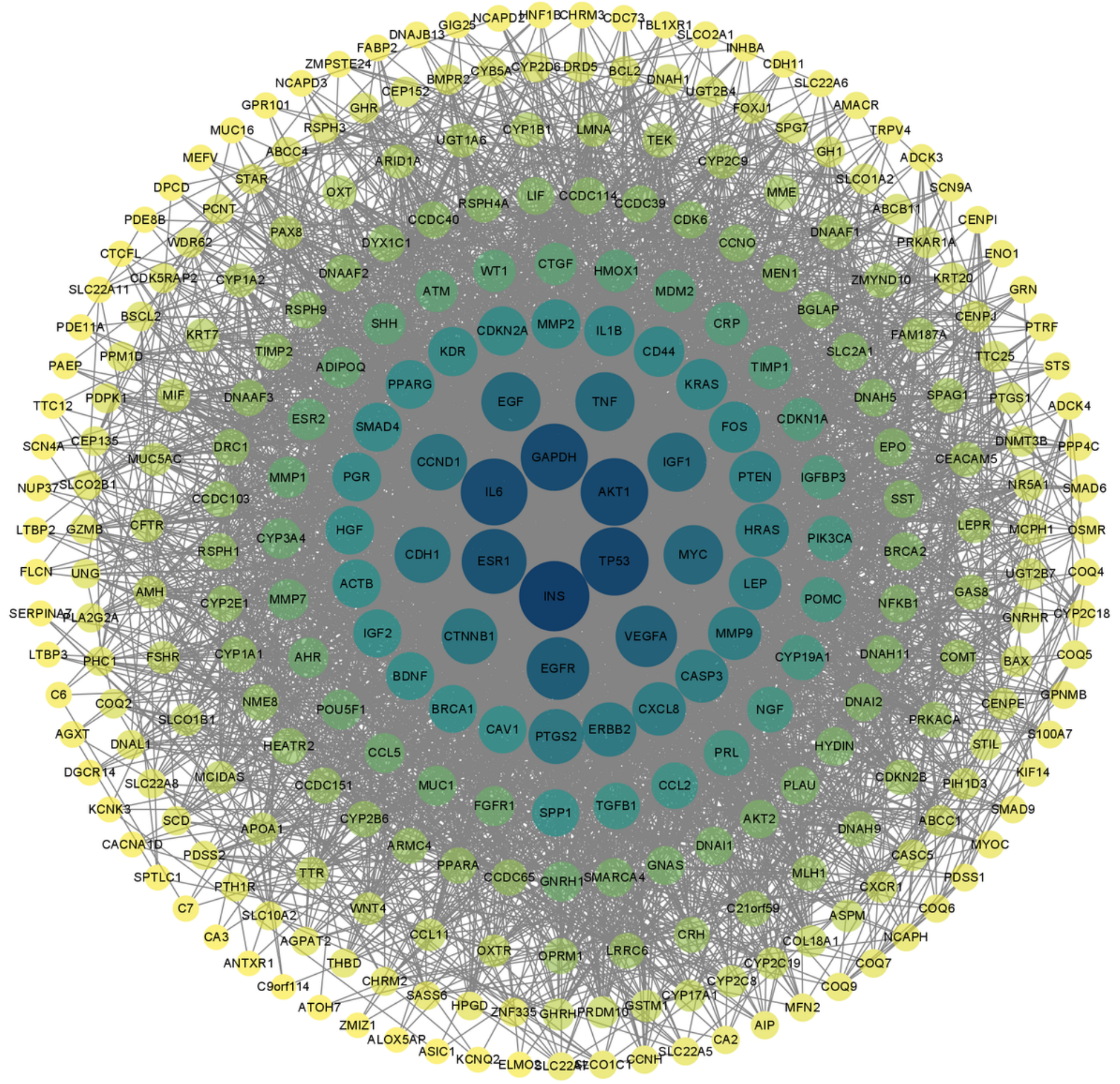

Figure 4

PPI network of PD targets. The sizes and colors of the nodes are illustrated from big to small and blue to yellow in descending order of degree values 
(a)

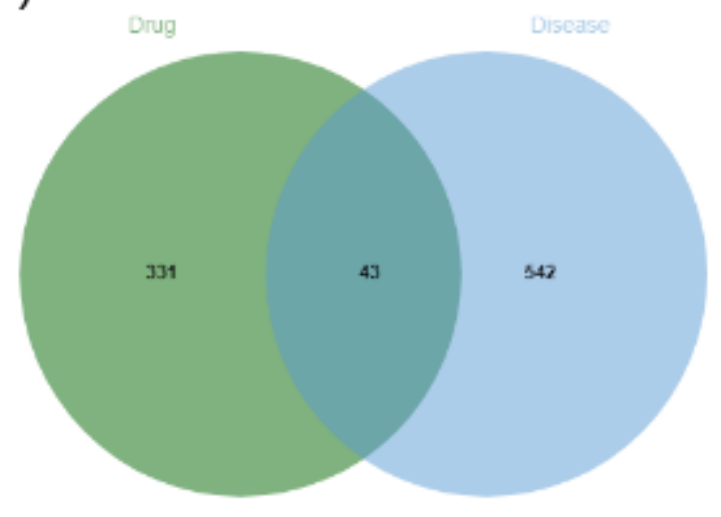

(b)

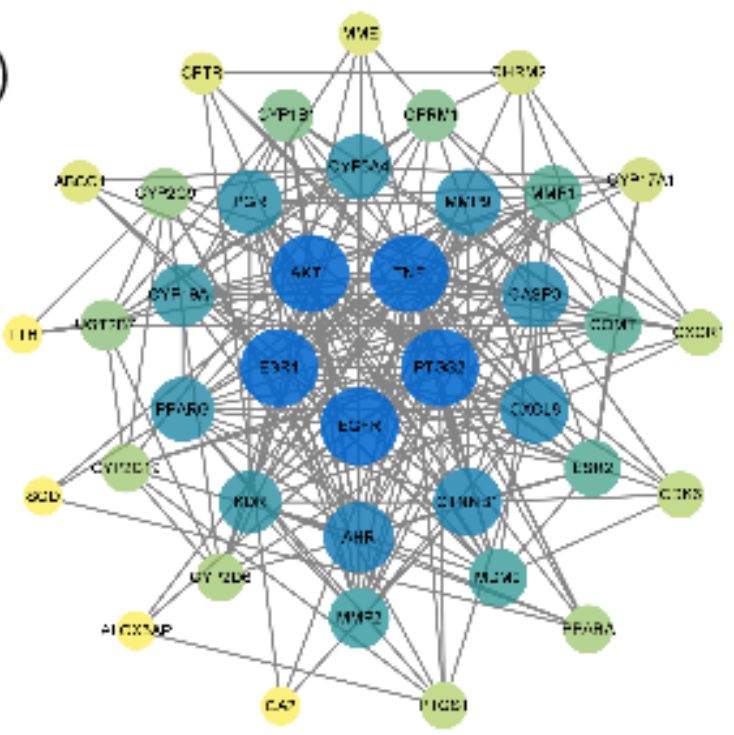

\section{Figure 5}

Venn diagram and PPI network of compound-PD targets. a.Venn diagram of intersecting targets of GFF and PD. b.PPI network of compound-PD targets. The sizes and colors of the nodes are illustrated from big to small and blue to yellow in descending order of degree values 


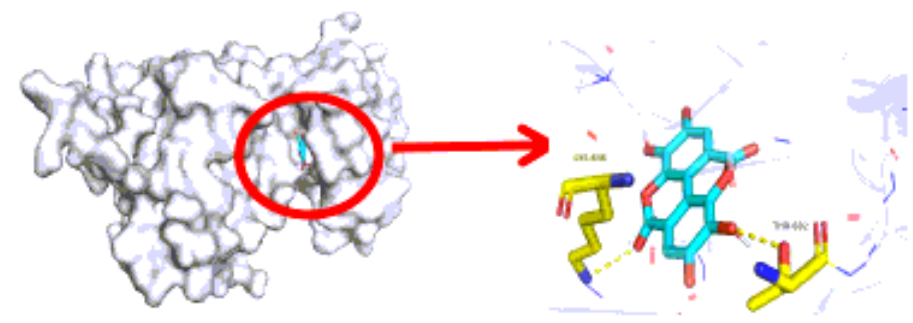

ellagic acid act on EGFR (Affinity=-9.4 kcal/mol)

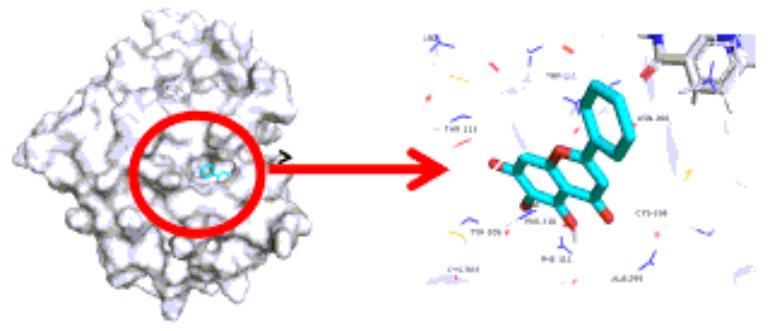

baicalein act on AHR(Affinity=-10.2kacal $/ \mathrm{mol}$ )
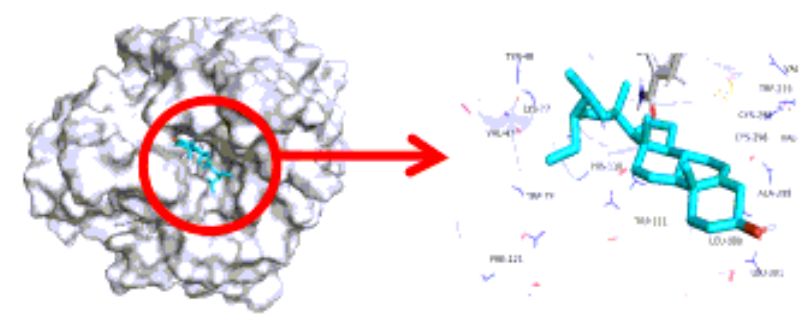

Stigmasterol act on CTNNB1(Affinity=-10.3kacal $/ \mathrm{mol}$ )

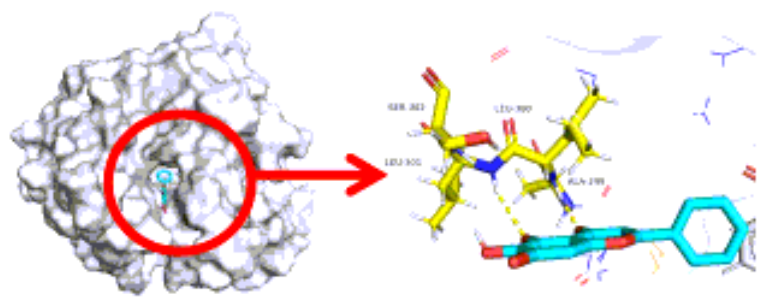

baicalein act on CTNNB1(Affinity=-9.4kacal $/ \mathrm{mol}$ )

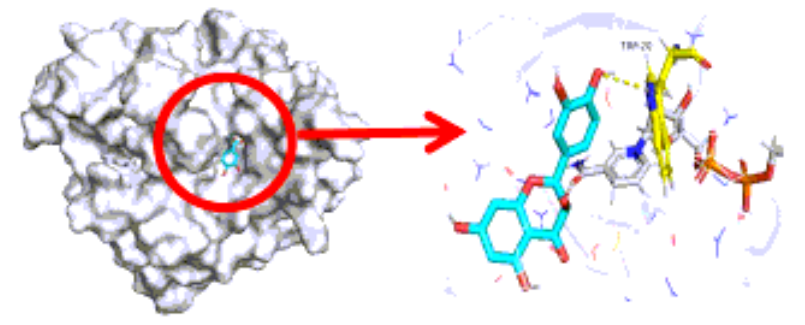

taxifolin act on AHR(Affinity=-10.3kacal $/ \mathrm{mol}$ )

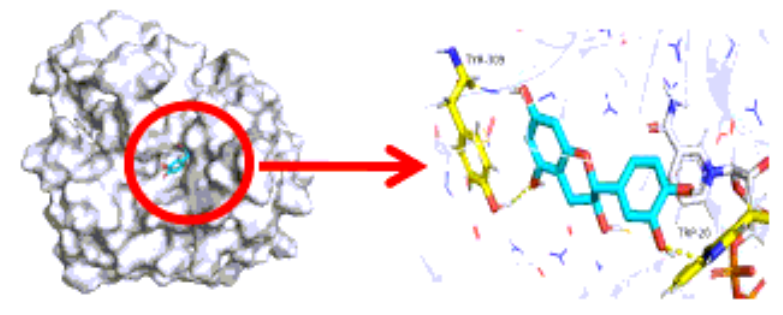

(+)-catechin act on AHR(Affinity=-10.2kacal $/ \mathrm{mol}$ )

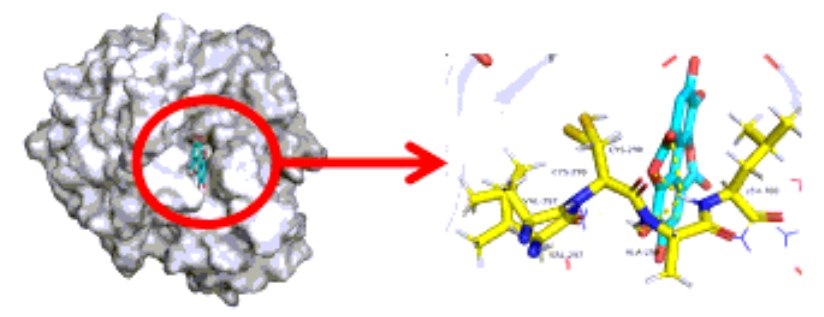

ellagic acid act on CTNNB1(Affinity $=-9.9 \mathrm{kacal} / \mathrm{mol}$ )

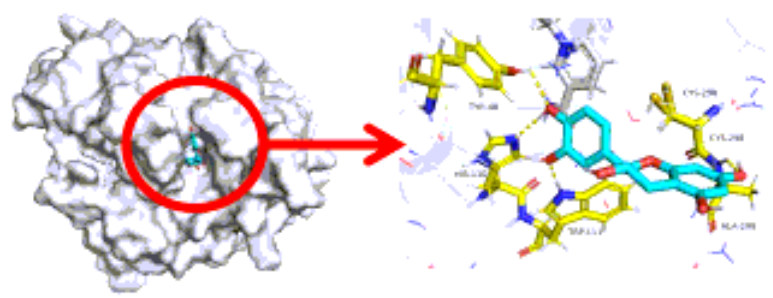

(+)-catechin act on CTNNB1(Affinity=-9.3kacal $/ \mathrm{mol}$ )

\section{Figure 6}

GO \& KEGG enrichment analysis. a GO enrichment analysis. BP: biological processes, CC:cell component, MF: molecular function. b KEGG pathway analysis. The sizes of the bubbles are illustrated from big to small in descending order of the number of the potential targets involved in the pathways. 


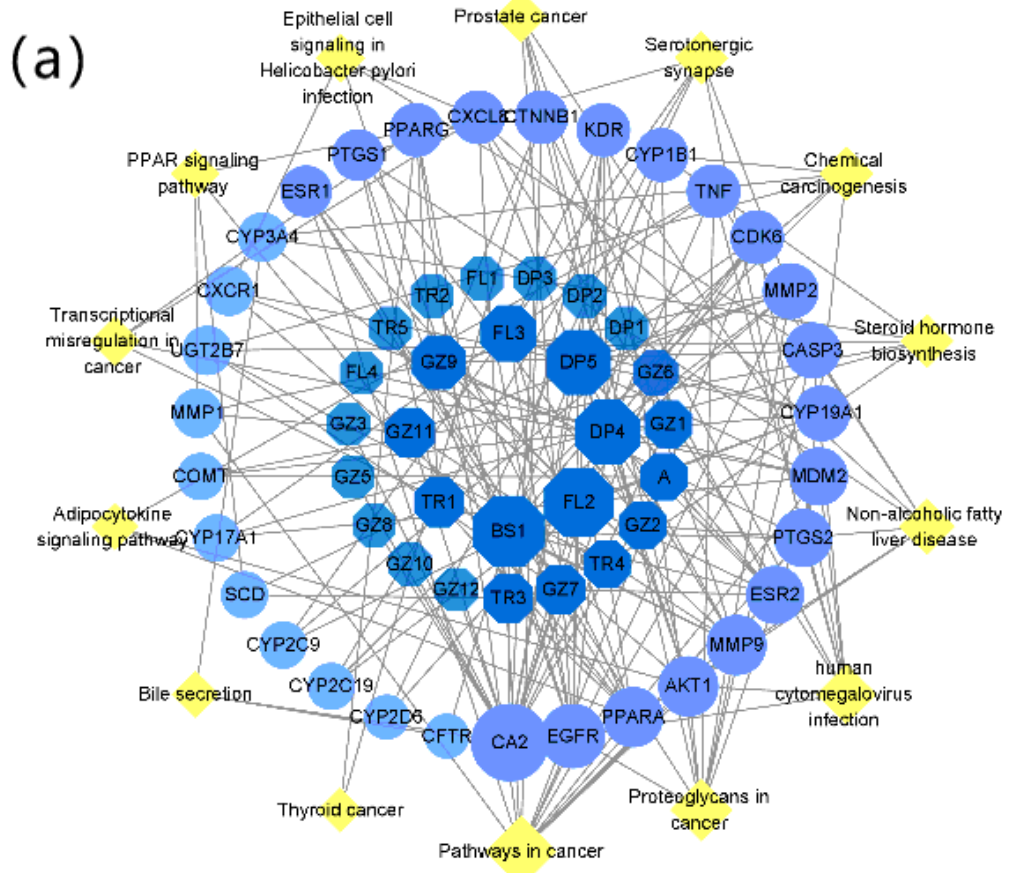

(b)

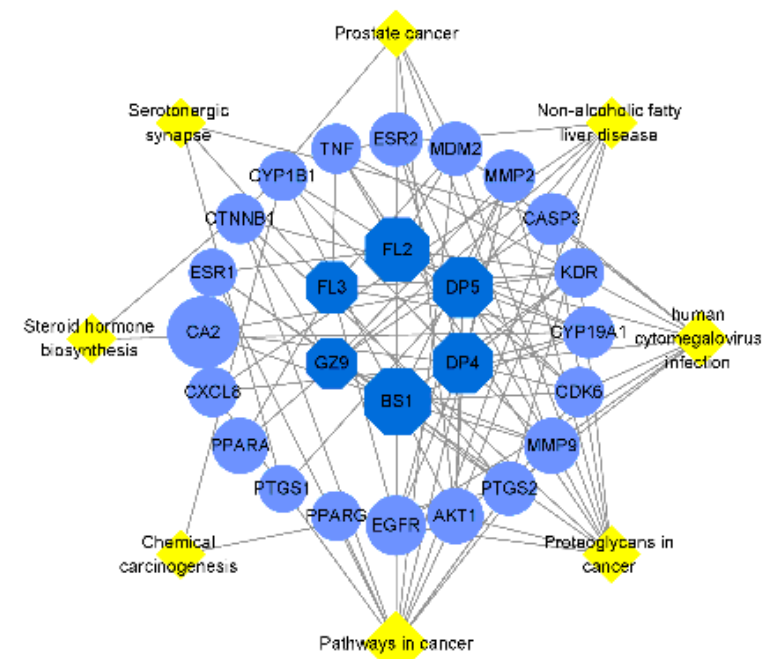

Figure 7

Analysis of target-compound docking simulation

\section{Supplementary Files}

This is a list of supplementary files associated with this preprint. Click to download.

- CTable11.png

- CTable12.png

- CTable13.png

- CTable14.png

- C.Fig.1.png 